\title{
Numerical simulation on the characteristics of smoke exhaust effect with different sets of smoke vent in deep buried inclined channel fire
}

\author{
Lu Yuhan ${ }^{1, *}$, Weng Miao cheng ${ }^{1,2,3}$, Liu Fang ${ }^{1,2,3}$ \\ ${ }^{1}$ School of Civil Engineering, Chongqing University, Chongqing, 400045, P.R. China; \\ ${ }^{2}$ Key Laboratory of New Technology for Construction of Cities in Mountain Area of Ministry of Education (Chongqing University), \\ Chongqing, 400045, P.R. China; \\ ${ }^{3}$ Joint International Research Laboratory of Green Buildings \& Built Environments, Chongqing, 400045, P.R. China;
}

\begin{abstract}
Deep buried metro stations require longer and more inclined exit passages to connect with the outside. The fire characteristics of these inclined and narrow passages are significantly different from those of above-ground or shallow buried metro station exit passages, and at the same time fires in those inclined channels have a greater risk. This paper takes a channel of deep buried tunnel station in Chongqing as an example and simplifies the actual passage to establish a 3D model to study the smoke spread characteristics along the passage with different smoke vent characteristics including shape and location by FDS, as well as temperature distribution characteristic under different working conditions. The results show that: after a fire, smoke will spread upwards rapidly under the action of thermal buoyancy, and mechanical smoke exhaust plays a certain role in controlling smoke, which is more obvious at the beginning of the fire; there are differences in the smoke exhaust efficiency of different smoke extraction openings shape, but the differences are small, and square smoke vents have a slightly better effect on smoke control than other shapes of smoke vents; changing the location of smoke outlets has a greater impact on the environment in the tunnel. When the smoke vent is located directly above the fire source, the mechanical smoke control effect is significantly better than other positions, and as the distance between smoke vent and fire source increases, the average temperature along the passage increases. At the same time, because of the pressure difference after the fire, the emergency staircase and the upstream of the fire source are basically unaffected by smoke.
\end{abstract}

\section{Introduction}

With the explosive growth of population, the traditional spatial spreading idea of urban expansion is no longer applicable for current development and the urban development needs to live in the wide frame of pluralism. On this premise, high-rise buildings, deep and shallow buried underground spaces have emerged. To relieve the enormous traffic pressure of ground transportation, mass transit railway transport has been greatly developed around the world in recent years.

Since the first urban railway was opened in China, by the end of 2019, 210 lines have opened for operation in 40 cities, including Shanghai, Beijing, Guangzhou, Nanjing, Wuhan, Chongqing, with a total mileage of $6,386.9 \mathrm{~km}$ [1]. The deep buried urban railway stations appeared in some cities due to the limitation of land resources. It can make full use of underground space, as a result, it requires a long and inclined channel between the station and the ground entrances. Once a fire occurs in the channel, the smoke spreads along the evacuation direction, and the smoke spreads upward more quickly under the action of thermal buoyancy. At the same time, smoke is often a major cause of death in fires, so the mechanical smoke exhaust in the long-inclined channel of deep buried underground station is very important. In this paper, the smoke exhaust efficiency of mechanical smoke exhaust under different smoke vent characteristics is explored, and may provide potential solutions for the smoke control system design in channels of deep buried station.

Shi Congling et al. [2] used numerical simulation to study the transverse flow and longitudinal spread of smoke between different deep buried island station fires, at the same time gave the recommended smoke exhaust volume and smoke exhaust mode. Xie Xuan [3] took the deep buried long tunnel of Chongqing as the object, proposed two ventilation programs of the intermediate air shaft and the track-top exhaust. Liu Sheng [4] took small-scale experiments to study the influence of the inclining angle on the wind speed generated by combustion, the pressure difference and then concluded that the larger the inclination angle, the larger the wind speed and the more obvious the trend of wind speed changed. Kai Zhu [5] discussed the effect of slope on the smoke backflow distances in blocked subway tunnels through small-scale experiment and numerical simulation, which concluded that the steeper the 
slope, the longer the backflow length, and obtained the relevant formula for calculating the backflow length.

Previous work has investigated the smoke spread under different situations by numerical simulations, small-scale experiments, etc. In those works, the platform fire was the main focus in deep buried metro stations with less attention on long passageways fires. At the same time, in the case of inclined tunnel fires, the main focus has been on the relationship between smoke spread characteristics and inclination angle which took little research about the shape and location of smoke vents.

Therefore, this paper takes a deeply buried metro station in Chongqing as an example, and investigates the influence of smoke vents with different shapes on smoke exhaust when a fire breaks out in a deeply buried channel by numerical simulation.

\section{Numerical simulation}

\subsection{Modify Software FDS and Establishment of Numerical Model}

The simulations are carried out using FDS (Fire Dynamic Simulator), a fluid dynamics software developed by NISTBFRL (Building and Fire Research Laboratory) for simulating fluid flow in fires, which solves the low Mach number N-S equation (1) of fire buoyancy driven by numerical calculation. The results are widely used in the fire pre-simulation, fire reconstruction and so on. The source code of FDS is open and the accuracy has been studied and verified by scholars, so the numerical simulation using FDS in this paper is of high reliability.

$\left\{\begin{array}{c}\rho\left(\frac{\partial u}{\partial t}+u \frac{\partial u}{\partial x}+v \frac{\partial u}{\partial y}+w \frac{\partial u}{\partial z}\right)=f_{x}-\frac{\partial P}{\partial x}+\mu\left(\frac{\partial^{2} u}{\partial x^{2}}+\frac{\partial^{2} u}{\partial y^{2}}+\frac{\partial^{2} u}{\partial z^{2}}\right) \\ \rho\left(\frac{\partial v}{\partial t}+u \frac{\partial v}{\partial x}+v \frac{\partial v}{\partial y}+w \frac{\partial v}{\partial z}\right)=f_{y}-\frac{\partial P}{\partial y}+\mu\left(\frac{\partial^{2} v}{\partial x^{2}}+\frac{\partial^{2} v}{\partial y^{2}}+\frac{\partial^{2} v}{\partial z^{2}}\right) \\ \rho\left(\frac{\partial w}{\partial t}+u \frac{\partial w}{\partial x}+v \frac{\partial w}{\partial y}+w \frac{\partial w}{\partial z}\right)=f_{z}-\frac{\partial P}{\partial z}+\mu\left(\frac{\partial^{2} w}{\partial x^{2}}+\frac{\partial^{2} w}{\partial y^{2}}+\frac{\partial^{2} w}{\partial z^{2}}\right)\end{array}\right.$

The model contains a total of three inclined channels and three resting platforms, and the fire source is placed on the second rest platform to simulate a luggage fire. The intensity of the fire source is generally taken to be $2 \sim 5 \mathrm{MW}$ when an ordinary luggage fire starts, and this simulation uses a fire source with a heat release rate of $2.5 \mathrm{MW}$, sets the ambient temperature at $20^{\circ} \mathrm{C}$ and simulation time is 400s. The specific fire scenario is shown in Table 1 . The mechanical ventilation was set up in accordance with the operational drawings, with exhaust wind speed of $2 \mathrm{~m} / \mathrm{s}$, the specific model is shown in Figure 1(a).

\subsection{Model Building and Fire Scenarios Setting}

In order to investigate the influence of the smoke vent with different shapes on the smoke evacuation efficiency, this paper took FDS to simulate the study. Simplified modeling of the exit passageway was carried out, ignoring the two curved sections in the actual tunnel. The channel model is $9 \mathrm{~m}$ wide, $4 \mathrm{~m}$ high, about $100 \mathrm{~m}$ long on the plane with an inclination angle of $25^{\circ}$, the central platform is connected to the evacuation stairs. At the same time, this channel has two exits which connected to the outside world, an exit is
$9 \times 4 \mathrm{~m}$ at the top of the channel and another is $2.7 \times 3 \mathrm{~m}$ at the top of the evacuation stairs.

The model contains a total of three inclined channels and three resting platforms, and the fire source is placed on the second rest platform to simulate a luggage fire. The intensity of the fire source is generally taken to be $2 \sim 5 \mathrm{MW}$ when an ordinary luggage fire starts, and this simulation uses a fire source with a heat release rate of $2.5 \mathrm{MW}$, sets the ambient temperature at $20^{\circ} \mathrm{C}$ and simulation time is $400 \mathrm{~s}$. The specific fire scenario is shown in Table 1. The mechanical smoke exhaust was set up in accordance with the operational drawings, with exhaust wind speed of $2 \mathrm{~m} / \mathrm{s}$, the specific model is shown in Figure 1(a).

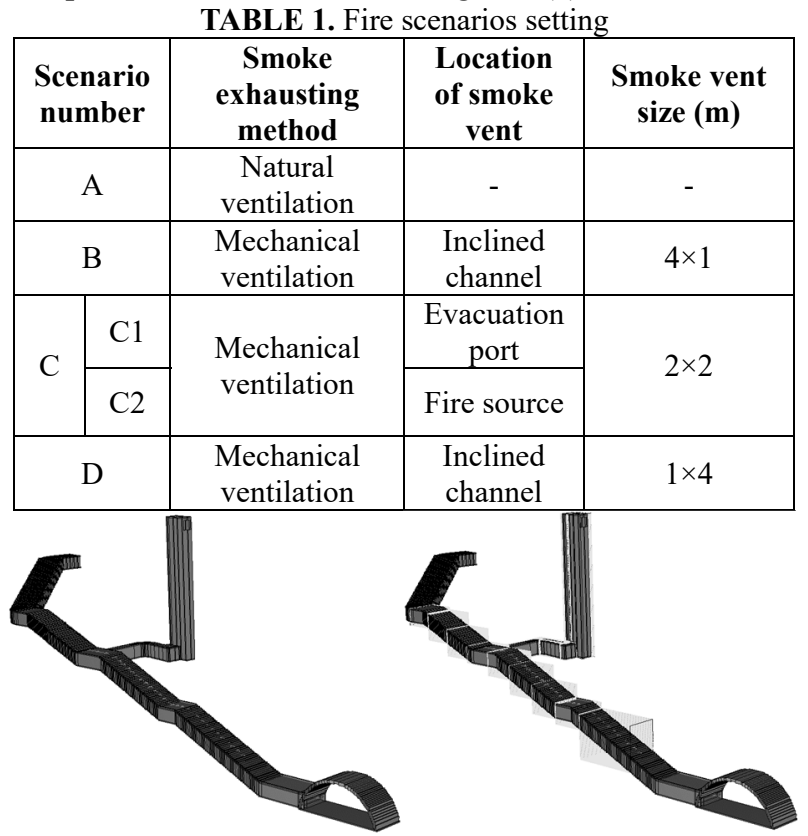

$\begin{array}{ll}\text { (a) the inclined channel } & \text { (b) grid setting of the model }\end{array}$ Fig.1 the channel model

To obtain the smoke flow and the distribution of each parameter during a fire in the passage, monitoring points and slices were arranged in the model. The airflow velocity and temperature were arranged in the channel and evacuation channel respectively. And we took a dense arrangement of monitoring points for the main research area, one set of measuring points were arranged for the stairway downstream of the fire source, the rest platform connected with the evacuation passage and the stairway upstream of the fire every $2 \mathrm{~m}$, and one set of measure points were arranged for the centre of each section at remaining locations. All measurement points are located in the centre line of the channel, as shown in Figure 2.

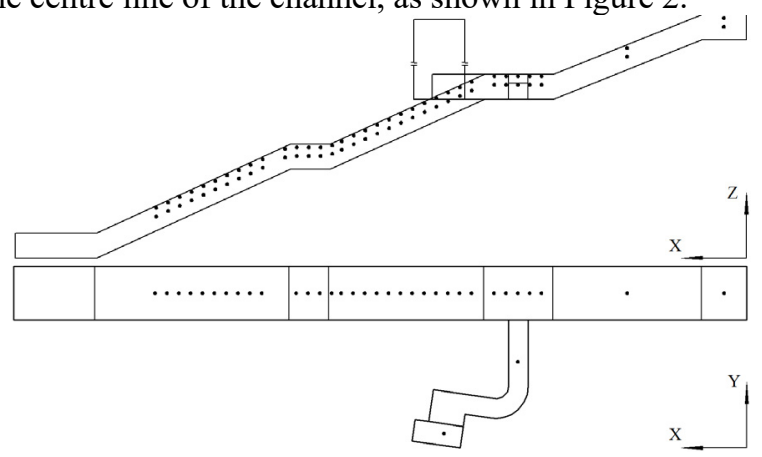

Fig.2 Monitoring points location 


\subsection{Grid Setting}

In the FDS simulating process, the accuracy of simulation results and simulation time are influenced by the size of the grid. The bulky grid can reduce the computing time, but the excessively coarse grid will greatly reduce the accuracy of calculation, and cause unreasonable oscillation. Therefore, it is necessary to select a reasonable grid size based on calculation accuracy, calculation time and computer performance, fire dynamics simulator User's Guide gives a reasonable mesh division method as shown in equation 2 .

$$
D^{*}=\left(\frac{Q}{\rho_{\infty} c_{p} T_{\infty} g^{0.5}}\right)^{\frac{2}{5}}
$$

some studies suggest that the grid between 0.1 and $0.2 \mathrm{~m}$ will improve simulation efficiency and minimize computational time while maintaining simulation accuracy. In order to reduce the calculation time while ensuring accurate simulation results, only the smoke spread area in the channel was meshed with a grid size of $0.25 \mathrm{~m}$. The model after meshing is shown in Figure 1(b).

\section{Results and analysis}

\subsection{Gas flow velocity}

Figure 3 gives longitudinal airflow velocity slices of four conditions at 100s, 200s, 300s and 400s.

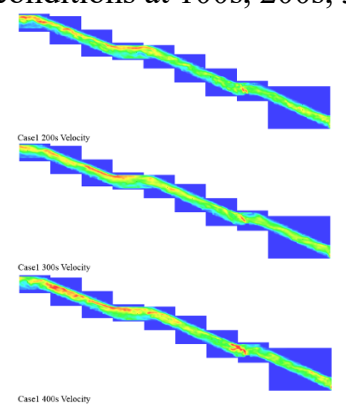

(a) Case $\mathrm{A}$

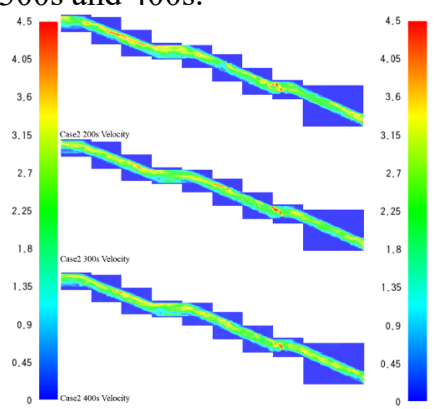

(b) Case B
Fig.3 flow velocity at different times

It can be seen that after the fire occurs, the smoke accumulates and spreads rapidly. Due to the thermal buoyancy, the smoke will spread rapidly upwards along the inclined channel, while it is difficult for the smoke flow to spread downwards under the action of the upstream wind pressure. And as the burning intensifies, the natural air supplement will increase which making little smoke accumulation in the upstream of fire, and the flame will incline to the downstream direction. At the same time, from the airflow velocity distribution are described in Figure 3 (a) and Figure 3 (b), it can be seen that the airflow velocity is the fastest under natural exhaust, and mechanical exhaust will weaken the overall airflow spreading velocity while exhausting the smoke.

\subsection{Temperature}

The roof longitudinal temperature distribution at different times for four scenarios are shown in Figure 4. The results show that as the distance from the fire source increases, the ceiling temperature keeps decreasing, the temperature drops significantly in the range of $5-10 \mathrm{~m}$ followed by a small fluctuation and then gradually stabilizes at around $50^{\circ} \mathrm{C}$. The maximum ceiling temperature stays within the range of $0-5 \mathrm{~m}$ from the fire. This is due to the smoke accumulates in the position towards the downstream under the action of thermal buoyancy and wind pressure caused by the upstream temperature, because of that the highest ceiling temperature does not appear directly above the fire source. It can be seen that under mechanical smoke exhaust, the stable temperature at the position far from the fire source is slightly lower than the natural ventilation working condition, the maximum ceiling temperature of mechanical ventilation is significantly lower than the maximum roof temperature of natural ventilation. Under different shapes smoke vents, the maximum roof temperature of $4 * 1$ smoke evacuation is the lowest.

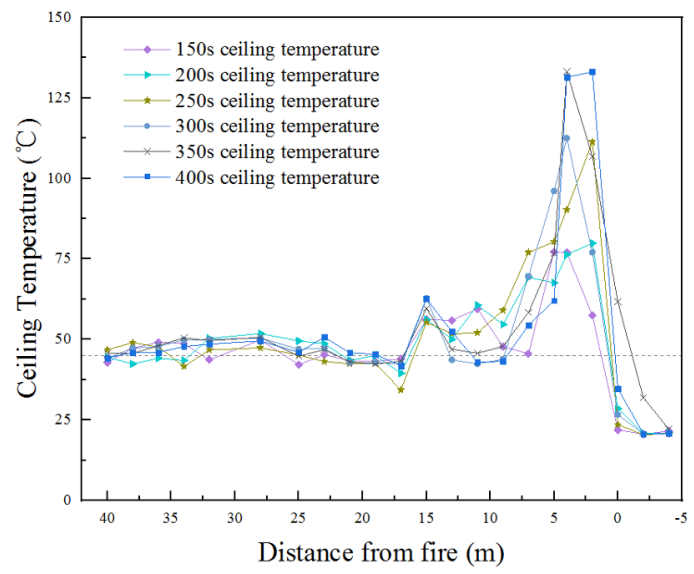

(a) Case B

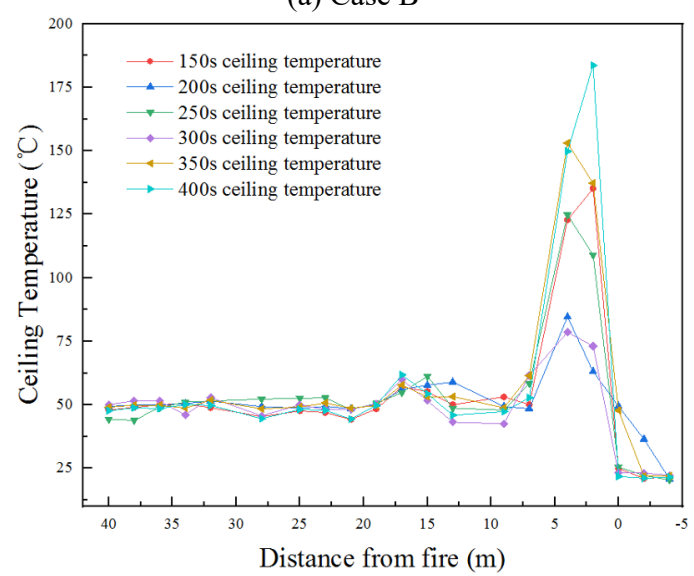

(b) Case D

Fig.4 ceiling longitudinal temperature distribution

Figure 5 shows that the longitudinal temperature distribution under the channel ceiling for the three scenarios with the vent located directly above the fire source, at the evacuation platform and the inclined channel respectively. It can be seen that the ceiling temperature has significant difference between different cases. When the smoke vent is above the fire source, the maximum ceiling temperature is significantly lower than the maximum temperature in the other case, and the temperature is also lower far away from the fire source, at this time mechanical ventilation has the strongest control over the smoke temperature in the inclined passage. 


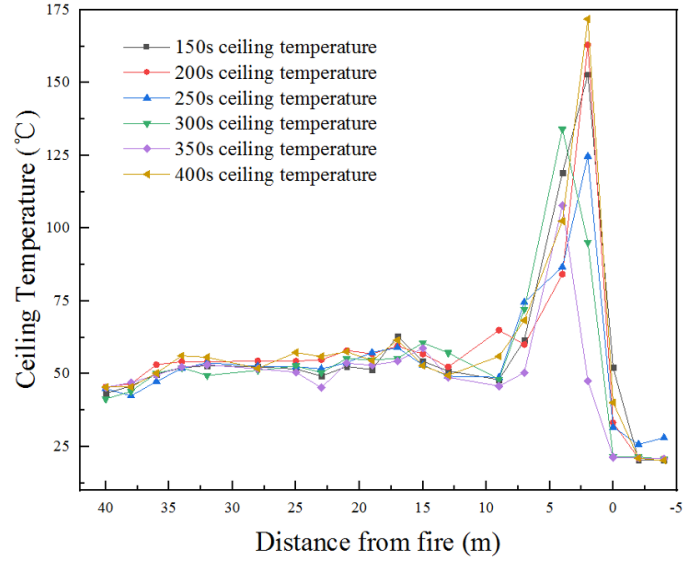

(a) Case $\mathrm{C} 1$

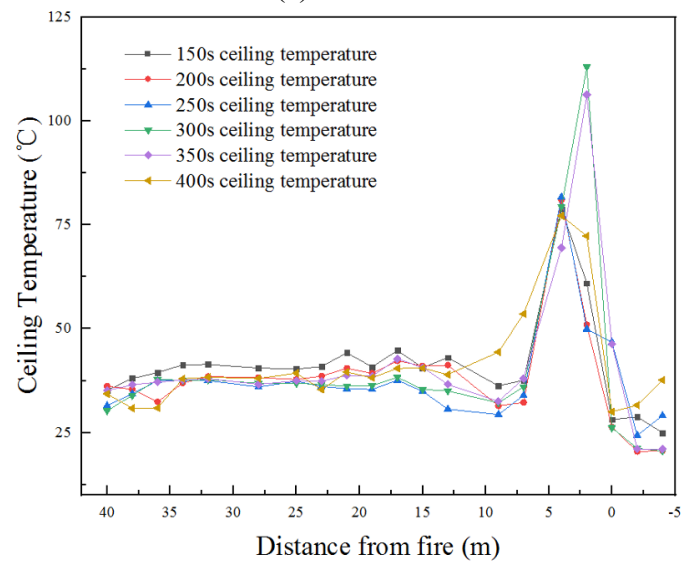

(b) Case $\mathrm{C} 2$

Fig.5 ceiling longitudinal temperature distribution

\section{Conclusion}

This paper investigated the influence of changing the shape and location of smoke vents on smoke movement, temperature in the inclined channel. A series of numerical simulations were taken and considered the changes of shape and location, the main conclusions are shown as follows:

(1) Through simulating the smoke exhaust effects of different vent shapes, it is found that when the smoke vent is a rectangle paralleling to the channel transversely, longitudinal temperature in the channel are lower than other shapes, but the difference is small, and the shape of the smoke vent is considered to have a small effect on the smoke control effect. In general, the smoke vent whose shape is the same as the vent in Case B plays an intercepting role for the smoke, and the smoke will surge into the vent under the action of thermal buoyancy; with the shortening of the long side of the smoke vent, the smoke on both sides of the vent cannot be inhaled and discharged in a timely manner, and the smoke will continue to spread and accumulate along the roof, at this time the smoke vent has a weaker intercepting role for the smoke in the same section, and the smoke discharge efficiency is reduced.

(2) Temperature distribution in the channel are vastly different under the change of distance between smoke vent and fire source. If the smoke vent is located directly above the fire source, the temperature in the inclined tunnel is significantly lower than other two cases. It is believed that this is due to the smoke plume being drawn directly into the smoke vent after the fire, and the exhaust absorbs in more smoke under the thermal buoyancy and the upward movement of the plume. Because of this, the amount of smoke spreading in the channel is reduced. As the distance between the smoke outlet and the fire source increases, the smoke exhaust effect weakens, and the lowest temperature along the path will appear at the smoke vent.

(3) When a fire breaks out in an inclined narrow channel, smoke will spread rapidly upwards along the inclined tunnel under the action of thermal buoyancy. The smoke spread direction is the same as the evacuation direction, it is important to control the smoke through considering reasonable smoke vents in mechanical smoke exhaust system. The change in smoke exhaust efficiency is small with the smoke vent shape changing and the difference is mainly reflected in the temperature. In practical engineering, similar inclined passages should use rectangular exhaust vents and pay attention to the direction of the long side of vents when designing, thus making the interception effect of smoke vents can be fully effective. At the same time, it is important identifying the source of the fire immediately and opening the smoke vent near the source in time.

\section{References}

1. China Urban Rail Transit Association, 2019. China Urban Rail Transit Market Development Report 2019

2. Ji, J. (2008) Studies on smoke movement and ventilation control mode in subway station fire. University of Science and Technology of China.

3. Dong, X.G. (2014) Numerical simulation of smoke exhaust effect in urban traffic link tunnel with longitudinal ventilation. Bejing University of Technology.

4. Xie, X. (2013) Numerical simulation study on fire ventilation of subway and deeply buried long tunnel. Southwest Jiantong University.

5. Wu, Y.J. (2013) Simulation study of influence factors on smoke control and extraction in high-rise building with corridor. Journal of Safety Science and Technology, 9: 36-42. 